\title{
Non-local boundary condition Steklov problem for a laplace equation in bounded domain
}

\author{
Aliev Nehan Ali ${ }^{1}$, Abbasova Aygun Khanlar ${ }^{1, *}$, Zeynalov Ramin M. ${ }^{2}$ \\ ${ }^{1}$ Baku State University, Baku, Azerbaijan \\ ${ }^{2}$ Institute of Cybernetics of Azerbaijan National Academy of Sciences, Baku, Azerbaijan
}

Email address:

aygun_abbasova@bk.ru (A. Aygun Khanlar), Ramin_z@rambler.ru (Z. Ramin M.)

To cite this article:

Aliev Nehan Ali, Abbasova Aygun Khanlar, Zeynalov Ramin M. . Non-local Boundary Condition Steklov Problem for A Laplace Equation in Bounded Domain, Science Journal of Applied Mathematics and Statistics. Vol. 1, No. 1, 2013, pp. 1-6.

doi: 10.11648/j.sjams.20130101.11

\begin{abstract}
In classic mathematical physics course, under the local boundary conditions the Dirichlet (first kind), Neuman (second kind) and at last special case of Poincare (third kind) boundary value problems were considered for a Laplace equation being a canonic form of elliptic type of equations. Later on for a Laplace equation, under non-local boundary condition the Steklov problem was investigated in [3] and a sufficient condition for Fredholm property was found. Note that here boundary conditions contains non -local and global terms and the investigation method consist of obtaining necessary conditions, regularization of them and reducing the stated boundary problem to the system of second kind Fredholm integral equation with non-singular kernel.
\end{abstract}

Keywords: Boundary Value Problem, Local, Non-Local And Global Boundary Condition, Steklov Problem, Necessary Conditions, Regularization, Fredholm Property

\section{Introduction}

It is known, that outside of geometrically symmetric domains for investigating the solution of boundary value problems, the potential theory is the most perfect method of mathematical physics equations [1], [2]. As the Green formula for a Laplace equation gives the harmonic function in the form of two summands, simple and double layer potentials, these potentials have been studied enough. We can say that not all the results obtained for these potentials have been found their application. So, the jump formulas for the double layer potential and for a normal derivative of a simple layer potential which arises during investigation the solutions of the Neumann and Dirichlet problems was in wide use.

In spite of the fact that the tangential derivative of a simple layer potential and the tangential and normal derivatives of the double layer potential have been investigated [1], they have not found their applications.

Our method of investigation is the continuation of the potential theory and makes it possible to look for the solution of a boundary value problem in the form given by the second Green formula [3]-[5]. Comparing the Cauchy problem for the wave equation with the boundary problem for the Laplas equation it should be noted that the functions participating in the D'Alambert formula obtained for the solution of the Cauchy problem are independent arbitrary functions (the functions given in the initial conditions), having given them we can get the solution of the Cauchy problem from D'Alambert formula [2]. However, in the second Green formula established for a Laplace equation, the harmonic function is obtained in the form of two summands, in one of them, a boundary value of the unknown function, in another one a boundary value of the normal derivative of this functions participates. These functions are not arbitrary functions as in the D'Alambert formula [2], they can't be given (for Laplace equation) arbitrarily. The relation between them are necessary conditions given by us.

The boundary value problems for elliptic (CauchyRiemann, Laplace), hyperbolic, mixed, composite type, Bitsadzeh and Sobolev equations and some other equations were considered by this method [3]-[11]. Note that Steklov problem, is generally considered for the second order elliptic type of equation with local boundary conditions. We began our researches of a Steklov problem with the elliptic type of equation of the first order, i.e. Cauchy-Riemann equation [8], [10]. Local boundary conditions of Dirichlet, Neumann, Poincare are unacceptable for this equation, therefore we considered a problem with not local boundary conditions. The presented work is devoted to research a Steklov problem (here spectral parameter is present only at boundary conditions) for Laplace equation where both local and global (i.e. integrals) terms are present at boundary 
conditions. The scheme of research belonging to us, absolutely differs from the methods of researches which are carried out for a Steklov problems with local boundary conditions.

One can find these papers in http/nihan.aliev.info.

\section{Problem Statement}

Let $D$ be a bounded, convex plane domain in the direction $x_{2}$, its boundary $\Gamma$ is a Lyapunov line [1]. If we project this domain along the axis $x_{1}$ parallel to $x_{2}$, the boundary $\Gamma$ will be divided into two parts. Denote these parts moving along $x_{2}$ by $\Gamma_{1}$ and $\Gamma_{2}$ and define these boundaries by equations $x_{2}=\gamma_{k}\left(x_{1}\right)$, and $x_{1} \in\left[a_{1}, b_{1}\right], k=1,2$ As the considered domain $D$ is convex in the direction $x_{2}$, the lines $\Gamma_{1}$ and $\Gamma_{2}$ will be expressed by one-valued functions $\gamma_{1}\left(x_{1}\right)$ and $\gamma_{2}\left(x_{1}\right)$.

Let's consider the boundary value problem

$$
\frac{\partial^{2} u(x)}{\partial x_{1}^{2}}+\frac{\partial^{2} u(x)}{\partial x_{2}^{2}}=0, x=\left(x_{1}, x_{2}\right) \in D \subset R^{2},
$$

$$
\left\{\begin{array}{l}
\left.\frac{\partial u(x)}{\partial x_{1}}\right|_{x_{2}=\gamma_{2}\left(x_{1}\right)}=\left.\beta\left(x_{1}\right) \frac{\partial u(x)}{\partial x_{1}}\right|_{x_{2}=\gamma_{1}\left(x_{1}\right)}+ \\
+\lambda \int_{1}^{b_{1}} K\left(x_{1}, \eta_{1}\right) u\left(\eta_{1}, \gamma_{1}\left(\eta_{1}\right)\right) d \eta_{1}, \\
\left.\frac{\partial u(x)}{\partial x_{2}}\right|_{x_{2}=\gamma_{2}\left(x_{1}\right)}=\left.\alpha\left(x_{1}\right) \frac{\partial u(x)}{\partial x_{2}}\right|_{x_{2}}=\gamma_{1}\left(x_{1}\right) \\
x_{1} \in\left[a_{1}, b_{1}\right]
\end{array}\right.
$$

where $K\left(x_{1}, \eta_{1}\right), \alpha\left(x_{1}\right), \beta\left(x_{1}\right)$ are complex-valued continuous functions, $\gamma_{1}\left(x_{1}\right)$ and $\gamma_{2}\left(x_{1}\right)$ are real-valued continuous functions, $\lambda$ is a spectral parameter. It is known that

$$
U(x-\xi)=\frac{1}{2 \pi} \ln |x-\xi|
$$

is a fundamental solution of two-dimensional Laplace equation [2].

\section{Basic Relations}

To obtain the first basic relation we get the second Green formula [1], [2] by means of Laplace equation and its fundamental solution (3). For this, multiply both hand sides of (1) by (3) and integrate with respect to domain $D$.

$$
\int_{D} \frac{\partial^{2} u(x)}{\partial x_{1}^{2}} U(x-\xi) d x+\int_{D} \frac{\partial^{2} u(x)}{\partial x_{2}^{2}} U(x-\xi) d x=0 .
$$

If we take the derivative by integration by parts from $u(x)$ to $U(x-\xi)$, we get:

$$
\begin{aligned}
& \int_{\Gamma}\left[u(x) \frac{\partial U(x-\xi)}{\partial x_{1}}-\frac{\partial u(x)}{\partial x_{1}} U(x-\xi)\right] \cdot \cos \left(v, x_{1}\right) d x+ \\
& \left.+\int_{\Gamma}^{[} u(x) \frac{\partial U(x-\xi)}{\partial x_{2}}-\frac{\partial u(x)}{\partial x_{2}} U(x-\xi)\right] \cdot \cos \left(v, x_{2}\right) d x= \\
& =\int_{D} u(x)\left[\frac{\partial^{2} U(x-\xi)}{\partial x_{1}^{2}}+\frac{\partial^{2} U(x-\xi)}{\partial x_{2}^{2}}\right] d x= \\
& =\left\{\begin{array}{l}
u(\xi), \xi \in D, \\
\frac{1}{2} u(\xi), \xi \in \Gamma,
\end{array}\right.
\end{aligned}
$$

here, we take into account that (3) is a fundamental solution of (1), i.e.

$$
\begin{gathered}
\frac{\partial^{2} U(x-\xi)}{\partial x_{1}^{2}}+\frac{\partial^{2} U(x-\xi)}{\partial x_{2}^{2}}=\delta(x-\xi), \text { so } \\
\delta(x-\xi)=\delta\left(x_{1}-\xi_{1}\right) \delta\left(x_{2}-\xi_{2}\right)
\end{gathered}
$$

is a two dimensional Dirac-delta function, $v$ is an external normal drawn to $\Gamma$. In order to get the remaining two basic relations, as in [3], [6], we multiply equation (1) by the first order derivatives of fundamental solution with respect to $x_{1}$ and $x_{2}$ and integrate the obtained expressions in domain $D$ :

$$
\int_{D} \frac{\partial^{2} u(x)}{\partial x_{1}^{2}} \cdot \frac{\partial U(x-\xi)}{\partial x_{1}} d x+\int_{D} \frac{\partial^{2} u(x)}{\partial x_{2}^{2}} \cdot \frac{\partial U(x-\xi)}{\partial x_{1}} d x=0,
$$

and

$$
\int_{D} \frac{\partial^{2} u(x)}{\partial x_{1}^{2}} \cdot \frac{\partial U(x-\xi)}{\partial x_{2}} d x+\int_{D} \frac{\partial^{2} u(x)}{\partial x_{2}^{2}} \cdot \frac{\partial U(x-\xi)}{\partial x_{2}} d x=0 .
$$

To get the analogy of the second Green formula from these expressions we conduct an integration by parts so that neither the third order derivatives of $u(x)$ and $U(x-\xi)$ on integrals on $D$ nor the second order derivatives could participate in integrals on $\Gamma$. Then we get:

$$
\begin{aligned}
& \int_{\Gamma}\left[\frac{\partial u(x)}{\partial x_{1}} \cdot \frac{\partial U(x-\xi)}{\partial x_{1}}-\frac{\partial u(x)}{\partial x_{2}} \cdot \frac{\partial U(x-\xi)}{\partial x_{2}}\right] \times \\
& +\int_{\Gamma}\left[\frac{\partial u(x)}{\partial x_{2}} \cdot \frac{\partial U(x-\xi)}{\partial x_{1}}+\frac{\partial u(x)}{\partial x_{1}} \cdot \frac{\partial U(x-\xi)}{\partial x_{2}}\right] \cdot \times \\
& \times \cos \left(v, x_{2}\right) d x=
\end{aligned}
$$




$$
\begin{aligned}
& =\int_{D} \frac{\partial u(x)}{\partial x_{1}}\left[\frac{\partial^{2} U(x-\xi)}{\partial x_{1}^{2}}+\frac{\partial^{2} U(x-\xi)}{\partial x_{2}^{2}}\right]= \\
& =\left\{\begin{array}{l}
\frac{\partial u(\xi)}{\partial \xi_{1}} \\
\frac{1}{2} \cdot \frac{\partial u(\xi)}{\partial \xi_{1}}, \xi \in \Gamma
\end{array}\right.
\end{aligned}
$$

In the same way, integrating by parts the second relation obtained from the above equation, similar to (5) we get:

$$
\begin{gathered}
\int_{\Gamma}^{[}\left[\frac{\partial u(x)}{\partial x_{1}} \cdot \frac{\partial U(x-\xi)}{\partial x_{2}}+\frac{\partial u(x)}{\partial x_{2}} \cdot \frac{\partial U(x-\xi)}{\partial x_{1}}\right] \cdot \cos \left(v, x_{1}\right) d x+ \\
+\int_{\Gamma}\left[\frac{\partial u(x)}{\partial x_{2}} \cdot \frac{\partial U(x-\xi)}{\partial x_{2}}-\frac{\partial u(x)}{\partial x_{1}} \cdot \frac{\partial U(x-\xi)}{\partial x_{1}}\right] \cdot \cos \left(v, x_{2}\right) d x= \\
=\int_{D} \frac{\partial u(x)}{\partial x_{2}}\left[\frac{\partial^{2} U(x-\xi)}{\partial x_{1}^{2}}+\frac{\partial^{2} U(x-\xi)}{\partial x_{2}^{2}}\right] d x= \\
=\left\{\begin{array}{l}
\frac{\partial u(\xi)}{\partial \xi_{2}}, \xi \in D, \\
\frac{1}{2} \cdot \frac{\partial u(\xi)}{\partial \xi_{2}}, \xi \in \Gamma .
\end{array}\right.
\end{gathered}
$$

so, we get the following statement:

Theorem 1. Let $D$ be a convex bounded domain in the direction $x_{2}$, its boundary $\Gamma=\bar{D} \backslash D$ is a Lyapunov line. Then any harmonic function determined on $D$ satisfies main relation (4)-(6).

\section{Necessary Conditions}

The second expressions from the basic relations (4)-(6) obtained above are necessary conditions. Separate them:

$$
\begin{aligned}
& \mathrm{u}\left(\xi_{1}, \gamma_{\mathrm{k}}\left(\xi_{1}\right)\right)=\frac{1}{\pi} \int_{\mathrm{a}_{1}}^{\mathrm{b}_{1}}\left[\mathrm{u}\left(\mathrm{x}_{1}, \gamma_{1}\left(\mathrm{x}_{1}\right)\right) \times\right. \\
& \frac{\mathrm{x}_{1}-\xi_{1}}{\left(\mathrm{x}_{1}-\xi_{1}\right)^{2}+\left(\gamma_{1}\left(\mathrm{x}_{1}\right)-\gamma_{\mathrm{k}}\left(\xi_{1}\right)\right)^{2}}-\left.\frac{\partial \mathrm{u}(\mathrm{x})}{\partial \mathrm{x}_{1}}\right|_{\mathrm{x}_{2}=\gamma_{1}\left(\mathrm{x}_{1}\right)} \\
& \left.\times \ln \sqrt{\left(\mathrm{x}_{1}-\xi_{1}\right)^{2}+\left(\gamma_{1}\left(\mathrm{x}_{1}\right)-\gamma_{\mathrm{k}}\left(\xi_{1}\right)\right)^{2}}\right] \cdot \gamma_{1}^{\prime}\left(\mathrm{x}_{1}\right) \mathrm{d} \mathrm{x}_{1} \\
& \frac{-\frac{1}{\pi} \int_{\mathrm{a}_{1}}^{\mathrm{b}_{1}}\left[\mathrm{u}\left(\mathrm{x}_{1}, \gamma_{2}\left(\mathrm{x}_{1}\right)\right) \times\right.}{\left(\mathrm{x}_{1}-\xi_{1}\right)^{2}+\left(\gamma_{2}\left(\mathrm{x}_{1}\right)-\gamma_{\mathrm{k}}\left(\xi_{1}\right)\right)^{2}}-\left.\frac{\partial \mathrm{u}(\mathrm{x})}{\partial \mathrm{x}_{1}}\right|_{\mathrm{x}_{2}=\gamma_{2}\left(\mathrm{x}_{1}\right)} \\
& \left.\times \ln \sqrt{\left(\mathrm{x}_{1}-\xi_{1}\right)^{2}+\left(\gamma_{2}\left(\mathrm{x}_{1}\right)-\gamma_{\mathrm{k}}\left(\xi_{1}\right)\right)^{2}}\right] \cdot \gamma_{2}^{\prime}\left(\mathrm{x}_{1}\right) \mathrm{dx} \mathrm{x}_{1}- \\
& \frac{1}{\pi} \int_{\mathrm{a}_{1}}^{\mathrm{b}_{1}}\left[\mathrm{u}\left(\mathrm{x}_{1}, \gamma_{1}\left(\mathrm{x}_{1}\right)\right) \times\right. \\
& \frac{\gamma_{1}\left(\mathrm{x}_{1}\right)-\gamma_{\mathrm{k}}\left(\xi_{1}\right)}{\left(\mathrm{x}_{1}-\xi_{1}\right)^{2}+\left(\gamma_{1}\left(\mathrm{x}_{1}\right)-\gamma_{\mathrm{k}}\left(\xi_{1}\right)\right)^{2}}-\left.\frac{\partial \mathrm{u}(\mathrm{x})}{\partial \mathrm{x}}\right|_{\mathrm{x}_{2}=\gamma_{1}\left(\mathrm{x}_{1}\right)}
\end{aligned}
$$

$$
\begin{aligned}
& \left.x \ln \sqrt{\left(x_{1}-\xi_{1}\right)^{2}+\left(\gamma_{1}\left(x_{1}\right)-\gamma_{k}\left(\xi_{1}\right)\right)^{2}}\right] \cdot d x_{1}+ \\
& \frac{1}{\pi} \int_{\mathrm{a}_{1}}^{\mathrm{b}_{1}}\left[\mathrm{u}\left(\mathrm{x}_{1}, \gamma_{2}\left(\mathrm{x}_{1}\right)\right) \times\right. \\
& \frac{\gamma_{2}\left(\mathrm{x}_{1}\right)-\gamma_{\mathrm{k}}\left(\xi_{1}\right)}{\left(\mathrm{x}_{1}-\xi_{1}\right)^{2}+\left(\gamma_{2}\left(\mathrm{x}_{1}\right)-\gamma_{\mathrm{k}}\left(\xi_{1}\right)\right)^{2}}-\left.\frac{\partial \mathrm{u}(\mathrm{x})}{\partial \mathrm{x}_{2}}\right|_{\mathrm{x}_{2}=\gamma_{2}\left(\mathrm{x}_{1}\right)} \times \\
& \left.\ln \sqrt{\left(\mathrm{x}_{1}-\xi_{1}\right)^{2}+\left(\gamma_{2}\left(\mathrm{x}_{1}\right)-\gamma_{\mathrm{k}}\left(\xi_{1}\right)\right)^{2}}\right] \mathrm{dx_{1 }} \text {, } \\
& \mathrm{k}=1,2 ; \xi_{1} \in\left[\mathrm{a}_{1}, \mathrm{~b}_{1}\right] \\
& \left.\frac{\partial u(\xi)}{\partial \xi_{1}}\right|_{\xi_{2}=\gamma_{k}\left(\xi_{1}\right)}=\frac{1}{\pi} \int_{a_{1}}^{b_{1}}\left[\left.\frac{\partial u(x)}{\partial x_{1}}\right|_{x_{2}=\gamma_{1}\left(x_{1}\right)} \times\right. \\
& \times \frac{x_{1}-\xi_{1}}{\left(x_{1}-\xi_{1}\right)^{2}+\left(\gamma_{1}\left(x_{1}\right)-\gamma_{k}\left(\xi_{1}\right)\right)^{2}}-\left.\frac{\partial u(x)}{\partial x_{2}}\right|_{x_{2}=\gamma_{1}\left(x_{1}\right)} \times \\
& \left.\cdot \times \frac{\gamma_{1}\left(x_{1}\right)-\gamma_{k}\left(\xi_{1}\right)}{\left(x_{1}-\xi_{1}\right)^{2}+\left(\gamma_{1}\left(x_{1}\right)-\gamma_{k}\left(\xi_{1}\right)\right)^{2}}\right] \cdot \gamma_{1}^{\prime}\left(x_{1}\right) d x_{1}- \\
& -\frac{1}{\pi} \int_{a_{1}}^{b_{1}}\left[\left.\frac{\partial u(x)}{\partial x_{1}}\right|_{x_{2}=\gamma_{2}\left(x_{1}\right)} \cdot \frac{x_{1}-\xi_{1}}{\left(x_{1}-\xi_{1}\right)^{2}+\left(\gamma_{2}\left(x_{1}\right)-\gamma_{k}\left(\xi_{1}\right)\right)^{2}}-\right.
\end{aligned}
$$$$
\left.-\left.\frac{\partial u(x)}{\partial x_{2}}\right|_{x_{2}=\gamma_{2}\left(x_{1}\right)} \cdot \frac{\gamma_{2}\left(x_{1}\right)-\gamma_{k}\left(\xi_{1}\right)}{\left(x_{1}-\xi_{1}\right)^{2}+\left(\gamma_{2}\left(x_{1}\right)-\gamma_{k}\left(\xi_{1}\right)\right)^{2}}\right] \cdot \gamma_{2}^{\prime}\left(x_{1}\right) d x-
$$$$
-\frac{1}{\pi} \int_{a_{1}}^{b_{1}}\left[\left.\frac{\partial u(x)}{\partial x_{2}}\right|_{x_{2}=\gamma_{1}\left(x_{1}\right)} \cdot \frac{x_{1}-\xi_{1}}{\left(x_{1}-\xi_{1}\right)^{2}+\left(\gamma_{1}\left(x_{1}\right)-\gamma_{k}\left(\xi_{1}\right)\right)^{2}}+\right.
$$$$
\left.+\left.\frac{\partial u(x)}{\partial x_{1}}\right|_{x_{2}=\gamma_{1}\left(x_{1}\right)} \cdot \frac{\gamma_{1}\left(x_{1}\right)-\gamma_{k}\left(\xi_{1}\right)}{\left(x_{1}-\xi_{1}\right)^{2}+\left(\gamma_{1}\left(x_{1}\right)-\gamma_{k}\left(\xi_{1}\right)\right)^{2}}\right] \cdot d x_{1}+
$$$$
+\frac{1}{\pi} \int_{a_{1}}^{b_{1}}\left[\left.\frac{\partial u(x)}{\partial x_{2}}\right|_{x_{2}=\gamma_{2}\left(x_{1}\right)} \frac{x_{1}-\xi_{1}}{\left(x_{1}-\xi_{1}\right)^{2}+\left(\gamma_{2}\left(x_{1}\right)-\gamma_{k}\left(\xi_{1}\right)\right)^{2}}+\right.
$$$$
\left.+\left.\frac{\partial u(x)}{\partial x_{1}}\right|_{x_{2}=\gamma_{2}\left(x_{1}\right)} \cdot \frac{\gamma_{2}\left(x_{1}\right)-\gamma_{k}\left(\xi_{1}\right)}{\left(x_{1}-\xi_{1}\right)^{2}+\left(\gamma_{2}\left(x_{1}\right)-\gamma_{k}\left(\xi_{1}\right)\right)^{2}}\right] \cdot d x_{1},
$$$$
k=1,2 ; \xi_{1} \in\left[a_{1}, b_{1}\right]
$$$$
\begin{aligned}
& \left.\frac{\partial u(\xi)}{\partial \xi_{2}}\right|_{\xi_{2}=\gamma_{k}\left(\xi_{1}\right)}=\frac{1}{\pi} \int_{a_{1}}^{b_{1}}\left[\left.\frac{\partial u(x)}{\partial x_{1}}\right|_{x_{2}=\gamma_{1}\left(x_{1}\right)} \times\right. \\
& \times \frac{\gamma_{1}\left(x_{1}\right)-\gamma_{k}\left(\xi_{1}\right)}{\left(x_{1}-\xi_{1}\right)^{2}+\left(\gamma_{1}\left(x_{1}\right)-\gamma_{k}\left(\xi_{1}\right)\right)^{2}}+\left.\frac{\partial u(x)}{\partial x_{2}}\right|_{x_{2}=\gamma_{1}\left(x_{1}\right)} \times \\
& \left.\times \frac{x_{1}-\xi_{1}}{\left(x_{1}-\xi_{1}\right)^{2}+\left(\gamma_{1}\left(x_{1}\right)-\gamma_{k}\left(\xi_{1}\right)\right)^{2}}\right] \cdot \gamma_{1}^{\prime}\left(x_{1}\right) d x_{1}-
\end{aligned}
$$ 


$$
\begin{aligned}
& \frac{1}{\pi} \int_{a_{1}}^{b_{1}}\left[\left.\frac{\partial u(x)}{\partial x_{1}}\right|_{x_{2}=\gamma_{2}\left(x_{1}\right)} \cdot \frac{\gamma_{2}\left(x_{1}\right)-\gamma_{k}\left(\xi_{1}\right)}{\left(x_{1}-\xi_{1}\right)^{2}+\left(\gamma_{2}\left(x_{1}\right)-\gamma_{k}\left(\xi_{1}\right)\right)^{2}}+\right. \\
& \left.+\left.\frac{\partial u(x)}{\partial x_{2}}\right|_{x_{2}=\gamma_{2}\left(x_{1}\right)} \frac{x_{1}-\xi_{1}}{\left(x_{1}-\xi_{1}\right)^{2}+\left(\gamma_{2}\left(x_{1}\right)-\gamma_{k}\left(\xi_{1}\right)\right)^{2}}\right] \times \\
& \times \gamma_{2}^{\prime}\left(x_{1}\right) d x_{1}-\frac{1}{\pi} \int_{a_{1}}^{b_{1}}\left[\left.\frac{\partial u(x)}{\partial x_{2}}\right|_{x_{2}=\gamma_{1}\left(x_{1}\right)} \times\right. \\
& \times \frac{\gamma_{1}\left(x_{1}\right)-\gamma_{k}\left(\xi_{1}\right)}{\left(x_{1}-\xi_{1}\right)^{2}+\left(\gamma_{1}\left(x_{1}\right)-\gamma_{k}\left(\xi_{1}\right)\right)^{2}}- \\
& \left.-\left.\frac{\partial u(x)}{\partial x_{1}}\right|_{x_{2}=\gamma_{1}\left(x_{1}\right)} \cdot \frac{x_{1}-\xi_{1}}{\left(x_{1}-\xi_{1}\right)^{2}+\left(\gamma_{1}\left(x_{1}\right)-\gamma_{k}\left(\xi_{1}\right)\right)^{2}}\right] d x_{1}+ \\
& +\frac{1}{\pi} \int_{a_{1}}^{b_{1}}\left[\left.\frac{\partial u(x)}{\partial x_{2}}\right|_{x_{2}=\gamma_{2}\left(x_{1}\right)} \frac{\gamma_{2}\left(x_{1}\right)-\gamma_{k}\left(\xi_{1}\right)}{\left(x_{1}-\xi_{1}\right)^{2}+\left(\gamma_{2}\left(x_{1}\right)-\gamma_{k}\left(\xi_{1}\right)\right)^{2}}-\right. \\
& -\left.\frac{\partial u(x)}{\partial x_{1}}\right|_{x_{2}=\gamma_{2}\left(x_{1}\right)} \times \\
& \left.\times \frac{x_{1}-\xi_{1}}{\left(x_{1}-\xi_{1}\right)^{2}+\left(\gamma_{2}\left(x_{1}\right)-\gamma_{k}\left(\xi_{1}\right)\right)^{2}}\right] \cdot d x_{1} \\
& k=1,2 ; \xi_{1} \in\left[a_{1}, b_{1}\right] .
\end{aligned}
$$

So we get the following statement:

Theorem 2. Under the conditions of theorem 1, any harmonic function determined in $D$ satisfies necessary conditions (7)-(9).

\section{Separation of Singularities}

Let consider expression (7) obtained for necessary conditions. Here at right side at $k=1$ first and fifth terms, and at $k=2$ third and seventh singular terms cancels each other, so there is no singularity in (7) and, therefore it is regular. Now, consider the second necessary conditions (8). The first, second, fifth and sixth expressions at its right side are singular at $k=1$. Here, the first and sixth terms cancels each other, and the second and fifth singular terms are combined, therefore, at $k=1$ from (8) we get:

$$
\begin{aligned}
& \left.\frac{\partial u(\xi)}{\partial \xi_{1}}\right|_{\xi_{2}=\gamma_{1}\left(\xi_{1}\right)}= \\
& =-\left.\frac{1}{\pi} \int_{a_{1}}^{b_{1}} \frac{\partial u(x)}{\partial x_{2}}\right|_{x_{2}=\gamma_{1}\left(x_{1}\right)} \cdot \frac{d x_{1}}{x_{1}-\xi_{1}}+\ldots, \quad \xi_{1} \in\left[a_{1}, b_{1}\right],
\end{aligned}
$$

where the dots indicates the sum of the obtained regular expressions. In the same way at $\mathrm{k}=2$ we get:

$$
\begin{aligned}
& \left.\frac{\partial u(\xi)}{\partial \xi_{1}}\right|_{\xi_{2}=\gamma_{2}\left(\xi_{1}\right)}=\left.\frac{1}{\pi} \int_{a_{1}}^{b_{1}} \frac{\partial u(x)}{\partial x_{2}}\right|_{x_{2}=\gamma_{2}\left(x_{1}\right)} \frac{d x_{1}}{x_{1}-\xi_{1}}+\ldots \\
& \xi_{1} \in\left[a_{1}, b_{1}\right] \text {. }
\end{aligned}
$$

At last consider the expression (9). Here at $k=1$ there is singularity in the second and fifth terms in the right side, which are mutually canceled. Other terms have no singularity. The obtained expression is as follows:

$$
\begin{aligned}
& \left.\frac{\partial u(\xi)}{\partial \xi_{2}}\right|_{\xi_{2}=\gamma_{1}\left(\xi_{1}\right)}=\left.\frac{1}{\pi} \int_{a_{1}}^{b_{1}} \frac{\partial u(x)}{\partial x_{1}}\right|_{x_{2}=\gamma_{1}\left(x_{1}\right)} \cdot \frac{d x_{1}}{x_{1}-\xi_{1}}+\ldots \\
& \xi_{1} \in\left[a_{1}, b_{1}\right] .
\end{aligned}
$$

In the same way, for $k=2$ we get:

$$
\left.\frac{\partial u(\xi)}{\partial \xi_{2}}\right|_{\xi_{2}=\gamma_{2}\left(\xi_{1}\right)}=-\left.\frac{1}{\pi} \int_{a_{1}}^{b_{1}} \frac{\partial u(x)}{\partial x_{1}}\right|_{x_{2}=\gamma_{2}\left(x_{1}\right)} \cdot \frac{d x_{1}}{x_{1}-\xi_{1}}+\ldots, \quad \xi_{1} \in\left[a_{1}, b_{1}\right] .
$$

where the dots indicates the sum of the regular expressions, which doesn't participate expressions $u\left(x_{1}, \gamma_{k}\left(x_{1}\right)\right)$. Thus, we see that each of the four necessary conditions given in (8) and (9) has singular summands.

\section{Regularization}

Set up the following combination by means of the expressions (10) and (11) obtained by the necessary conditions above:

$$
\begin{gathered}
\left.\frac{\partial u(\xi)}{\partial \xi_{1}}\right|_{\xi_{2}=\gamma_{2}\left(\xi_{1}\right)}+\left.\alpha\left(\xi_{1}\right) \frac{\partial u(\xi)}{\partial \xi_{1}}\right|_{\xi_{2}=\gamma_{1}\left(\xi_{1}\right)}=\frac{1}{\pi} \int_{a_{1}}^{b_{1}}\left[\left.\frac{\partial u(x)}{\partial x_{2}}\right|_{x_{2}=\gamma_{2}\left(x_{1}\right)}-\right. \\
\left.-\left.\alpha\left(\xi_{1}\right) \frac{\partial u(x)}{\partial x_{2}}\right|_{x_{2}=\gamma_{1}\left(x_{1}\right)}\right] \cdot \frac{d x_{1}}{x_{1}-\xi_{1}}+\ldots= \\
=\frac{1}{\pi} \int_{a_{1}}^{b_{1}}\left[\left.\frac{\partial u(x)}{\partial x_{2}}\right|_{x_{2}=\gamma_{2}\left(x_{1}\right)}-\left.\alpha\left(x_{1}\right) \frac{\partial u(x)}{\partial x_{2}}\right|_{x_{2}=\gamma_{1}\left(x_{1}\right)}\right] \frac{d x_{1}}{x_{1}-\xi_{1}}+\ldots=\ldots \\
\xi_{1} \in\left[a_{1}, b_{1}\right] .
\end{gathered}
$$

So, we used the second of boundary conditions (2) for obtaining the regular relation (14). Now, similar to (14), set up the following expression by means of (12) and (13):

$$
\begin{aligned}
& \left.\quad \frac{\partial u(\xi)}{\partial \xi_{2}}\right|_{\xi_{2}=\gamma_{2}\left(\xi_{1}\right)}+\left.\beta\left(\xi_{1}\right) \frac{\partial u(\xi)}{\partial \xi_{2}}\right|_{\xi_{2}=\gamma_{1}\left(\xi_{1}\right)}= \\
& =-\frac{1}{\pi} \int_{a_{1}}^{b_{1}}\left[\left.\frac{\partial u(x)}{\partial x_{1}}\right|_{x_{2}=\gamma_{2}\left(x_{1}\right)}-\left.\beta\left(\xi_{1}\right) \frac{\partial u(x)}{\partial x_{1}}\right|_{x_{2}=\gamma_{1}\left(x_{1}\right)}\right] \times \\
& \quad \times \frac{d x_{1}}{x_{1}-\xi_{1}}+\ldots=-\frac{\lambda}{\pi} \int_{a_{1}}^{b_{1}} \frac{d x_{1}}{x_{1}-\xi_{1}} \times \\
& \quad \times \int_{a_{1}}^{b_{1}} K\left(x_{1}, \eta_{1}\right) u\left(\eta_{1}, \gamma_{1}\left(\eta_{1}\right)\right) d \eta_{1}+\ldots
\end{aligned}
$$

In the singular integral in the right hand side of the obtained (15) we change the turn of integrals and regularize it. Then, we get the following regular expression:

$$
\begin{aligned}
& \left.\frac{\partial u(\xi)}{\partial \xi_{2}}\right|_{\xi_{2}=\gamma_{2}\left(\xi_{1}\right)}+\left.\beta\left(\xi_{1}\right) \frac{\partial u(\xi)}{\partial \xi_{2}}\right|_{\xi_{2}=\gamma_{1}\left(\xi_{1}\right)}= \\
& =-\frac{\lambda}{\pi} \int_{a_{1}}^{b_{1}} u\left(\eta_{1}, \gamma_{1}\left(\eta_{1}\right)\right) d \eta_{1} \int_{a_{1}}^{b_{1}} K\left(x_{1}, \eta_{1}\right) \frac{d x_{1}}{x_{1}-\xi_{1}}+\ldots
\end{aligned}
$$


Thus, if we combine the regular expressions (14), (16) with boundary conditions (2), we get the following four relations for boundary values of first order derivative:

$$
\begin{aligned}
& \left.\frac{\partial u(\xi)}{\partial \xi_{1}}\right|_{\xi_{2}=\gamma_{2}\left(\xi_{1}\right)}-\left.\beta\left(\xi_{1}\right) \frac{\partial u(\xi)}{\partial \xi_{1}}\right|_{\xi_{2}=\gamma_{1}\left(\xi_{1}\right)}= \\
& =\lambda \int_{a_{1}}^{b_{1}} K\left(\xi_{1}, \eta_{1}\right) u\left(\eta_{1}, \gamma_{1}\left(\eta_{1}\right)\right) d \eta_{1}, \\
& \left.\frac{\partial u(\xi)}{\partial \xi_{2}}\right|_{\xi_{2}=\gamma_{2}\left(\xi_{1}\right)}-\left.\alpha\left(\xi_{1}\right) \frac{\partial u(\xi)}{\partial \xi_{2}}\right|_{\xi_{2}=\gamma_{1}\left(\xi_{1}\right)}=0, \\
& \left.\frac{\partial u(\xi)}{\partial \xi_{1}}\right|_{\xi_{2}=\gamma_{2}\left(\xi_{1}\right)}+\left.\alpha\left(\xi_{1}\right) \frac{\partial u(\xi)}{\partial \xi_{1}}\right|_{\xi_{2}=\gamma_{1}\left(\xi_{1}\right)}=\ldots, \\
& \left.\frac{\partial u(\xi)}{\partial \xi_{2}}\right|_{\xi_{2}=\gamma_{2}\left(\xi_{1}\right)}+\left.\beta\left(\xi_{1}\right) \frac{\partial u(\xi)}{\partial \xi_{2}}\right|_{\xi_{2}=\gamma_{1}\left(\xi_{1}\right)}= \\
& =\frac{-\lambda}{\pi} \int_{a_{1}}^{b_{1}} u\left(\eta_{1}, \gamma_{1}\left(\eta_{1}\right)\right) d \eta_{1} \int_{a_{1}}^{b_{1}} K\left(x_{1}, \eta_{1}\right) \frac{d x_{1}}{x_{1}-\xi_{1}}+\ldots
\end{aligned}
$$

\section{Fredholm Property}

If we subtract the third equation from the first equation and the second equation from the fourth equation of (17) and accept the condition

$$
\alpha\left(x_{1}\right)+\beta\left(x_{1}\right) \neq 0,
$$

we get:

$$
\begin{gathered}
\left.\frac{\partial u(\xi)}{\partial \xi_{1}}\right|_{\xi_{2}=\gamma_{1}\left(\xi_{1}\right)}=-\frac{\lambda}{\alpha\left(\xi_{1}\right)+\beta\left(\xi_{1}\right)} \times \\
\quad \times \int_{a_{1}}^{b_{1}} K\left(\xi_{1}, \eta_{1}\right) u\left(\eta_{1}, \gamma_{1}\left(\eta_{1}\right)\right) d \eta_{1}+\ldots, \\
\left.\frac{\partial u(\xi)}{\partial \xi_{1}}\right|_{\xi_{2}=\gamma_{2}\left(\xi_{1}\right)}=-\frac{\lambda \alpha\left(\xi_{1}\right)}{\alpha\left(\xi_{1}\right)+\beta\left(\xi_{1}\right)} \times \\
\quad \times \int_{a_{1}}^{b_{1}} K\left(\xi_{1}, \eta_{1}\right) u\left(\eta_{1}, \gamma_{1}\left(\eta_{1}\right)\right) d \eta_{1}+\ldots \\
\left.\frac{\partial u(\xi)}{\partial \xi_{2}}\right|_{\xi_{2}=\gamma_{1}\left(\xi_{1}\right)}=-\frac{\lambda}{\pi\left(\alpha\left(\xi_{1}\right)+\beta\left(\xi_{1}\right)\right)} \times \\
\times \int_{a_{1}}^{b_{1}} u\left(\eta_{1}, \gamma_{1}\left(\eta_{1}\right)\right) d \eta_{1} \int_{a_{1}}^{b_{1}} K\left(x_{1}, \eta_{1}\right) \frac{d x_{1}}{x_{1}-\xi_{1}}+\ldots, \\
\left.\frac{\partial u(\xi)}{\partial \xi_{2}}\right|_{\xi_{2}=\gamma_{2}\left(\xi_{1}\right)}=-\frac{\lambda \alpha\left(\xi_{1}\right)}{\pi\left(\alpha\left(\xi_{1}\right)+\beta\left(\xi_{1}\right)\right)} \times \\
\times \int_{a_{1}}^{b_{1}} u\left(\eta_{1}, \gamma_{1}\left(\eta_{1}\right)\right) d \eta_{1} \int_{a_{1}}^{b_{1}} K\left(x_{1}, \eta_{1}\right) \frac{d x_{1}}{x_{1}-\xi_{1}}+\ldots .
\end{gathered}
$$

Thus, we obtained a system of four normal second order Fredholm integral equations for the boundary values of first order derivatives in the form (19). Finally, we combine system (19) with regular necessary conditions (7) obtained for $u\left(\xi_{1}, \gamma_{k}\left(\xi_{1}\right)\right) \quad k=1,2$ and get the system of second kind fredholm type homogeneous equations with weak singularity in the kernel, with respect to the six unknowns

$$
u\left(\xi_{1}, \gamma_{k}\left(\xi_{1}\right)\right),\left.\frac{\partial u(\xi)}{\partial \xi_{1}}\right|_{\substack{\xi_{2}=\gamma_{k}\left(\xi_{1}\right) \\ k=1,2}},\left.\frac{\partial u(\xi)}{\partial \xi_{2}}\right|_{\xi_{2}=\gamma_{k}\left(\xi_{1}\right)}
$$

At last, we get the following statement:

Theorem 3. Under the conditions of theorem 1, if $K\left(x_{1}, \eta_{1}\right)$ are continuous, $\alpha\left(x_{1}\right)$ and $\beta\left(x_{1}\right)$ are from the Hölder class, condition (18) is satisfied, then problem $(1),(2)$ is of Fredholm, i.e. the given boundary value problem is reduced to the system of second kind Fredholm type integral equations. This system has no singularity in the kernel.

\section{Unsolved Problems}

1) Assume that $D$ is a convex domain in the direction $x_{3}$ of three-dimensional space, the $\Gamma=\bar{D} \backslash D$ boundary is a Lyapunov plane. If we project this domain to the surface $x^{\prime}=\left(x_{1}, x_{2}\right)$ parallel to $x_{3}$, the boundary $\Gamma$ is divided into two parts. Denoting them by $\Gamma_{1}$ and $\Gamma_{2}$, respectively, we can express these planes by the following equations:

$$
\begin{gathered}
x_{3}=\gamma_{k}\left(x^{\prime}\right), k=1,2 \\
x^{\prime} \in S=\underset{\left(x_{1}, x_{2}\right)}{\operatorname{pr} . \Gamma_{1}}=\underset{\left(x_{1}, x_{2}\right)}{\operatorname{pr} . \Gamma_{2}}=\underset{\left(x_{1}, x_{2}\right)}{\operatorname{pr} . D} .
\end{gathered}
$$

We can investigate the following Steklov problem:

$$
\begin{gathered}
\Delta u(x) \equiv \sum_{k=1}^{3} \frac{\partial^{2} u(x)}{\partial x_{k}^{2}}=0, \\
x=\left(x_{1}, x_{2} x_{3}\right) \in D \subset R^{3} . \\
\sum_{k=1}^{3}\left[\left.\alpha_{j k}\left(x^{\prime}\right) \frac{\partial u(x)}{\partial x_{k}}\right|_{x_{3}}=\gamma_{1}\left(x^{\prime}\right)+\beta_{j k}\left(x^{\prime}\right) \times\right. \\
\left.\times\left.\frac{\partial u(x)}{\partial x_{k}}\right|_{x_{3}=\gamma_{2}\left(x^{\prime}\right)}\right]+\alpha_{j}\left(x^{\prime}\right) u\left(x^{\prime}, \gamma_{1}\left(x^{\prime}\right)\right)+\beta_{j}\left(x^{\prime}\right) \times \\
\times u\left(x^{\prime}, \gamma_{2}\left(x^{\prime}\right)\right)+\lambda \int_{S} K_{j}\left(x^{\prime}, \xi^{\prime}\right) u\left(\xi^{\prime}, \gamma_{j}\left(\xi^{\prime}\right)\right) d \xi^{\prime}=0 \\
j=1,2 ; x^{\prime} \in S,
\end{gathered}
$$

here $\lambda \in C$ is a spectral parameter, $K_{j}\left(x^{\prime}, \xi^{\prime}\right)$, $\alpha_{j k}\left(x^{\prime}\right) \beta_{j k}\left(x^{\prime}\right), \alpha_{j}\left(x^{\prime}\right), \beta_{j}\left(x^{\prime}\right)$ are the given sufficiently smooth functions.

2). The obtained problem has not been considered when the integral term in the boundary condition of the given problem is replaced by $\lambda u\left(x^{\prime}, \gamma_{j}\left(x^{\prime}\right)\right)$.

3). As a global term the integral $\int_{D} A_{j}\left(x^{\prime}, \xi\right) u(\xi) d \xi$ may be added to the boundary conditions of the given problem, so that the given boundary conditions are not linear dependent.

4). In two-dimensional case, for the Laplace equation the Steklov problem has not been considered under the boundary conditions containing non-local and global terms in multi-connected domain. 
5). In the same way, in two-dimensional case the Steklov problem remains open for convex domain.

\section{References}

[1] Courant R., Partial Equations.,M., "Mir" publishing house, $1964,830 \mathrm{pp}$.

[2] Vladimirov V.S., Equations of mathematical physics. M., "Nauka" publishing house, 1981, 512 pp.

[3] Aliev N.A., Suleymanov N. Investigation of solutions of boundary value problems containing a parameter in the boundary condition. Numerical methods to boundary value problems., (subject collection of scientific papers) Azerbaijan. University., Baku, 1989, p. 3-5.

[4] Aliev N. and Jahanshahi M., Solution of Poisson's equation with qlobal, local and non-local boundary conditions., International Journal of Mathematical Education in Science and Technology, vol 33 (2002), №2, pp.241-247.

[5] Bahrami, F., Aliev, N., Hosseini,, S.M., A Method for the reduction of four dimensional mixed problems with general boundary conditions to a system of second kind Fredholm inteqral equations. Italian Journal of pure and applied Mathematics, №17 (January 2005), pp.91-104.

[6] Aliev, N., Rezapour, Sh., Jahanshahi, M., A mixed problem for Navier-Stokes System, Mathematica Moravica Journal of University of Kragujevac, Serbia vol. 12-2 (2008), pp. 114.

[7] Aliev N.A, Abbasova A.Kh., The new approach to boundary problems for Cauchy-Riemann equation., News of Baku University, Series of Physico-Mathematical sciences, Azerbaijan, Baku,Vol.2, 2010, pp. 49-53.

[8] Abbasova A.Kh., Aliev N.A., About the Stefan problem for the Cauchy-Riemann equation with non-local and global terms in the boundary conditions., Material's International conference on mathematical theories and problems of their application and teaching is dedicated to the 870-th anniversary of great poet and philosopher Nizami Ganjavi, Azerbaijan, Ganja, September 23-25, 2011,pp.45-48.

[9] Abbasova A.Kh., Aliev N.A., Boundary problem on stripe with curvilinear boundaries., Journal of Contemporary Applied Mathematics, Vol.1, Issue 2, pp.67-71, December, 2011.

[10] Aliev N.A., Zeynalov R.M., Steklov problem for a first order elliptic type of equation. / News of Baku University, Series of Physico-Mathematical sciences, Azerbaijan, Baku,Vol.2, 2012,pp.13-21..

[11] Abbasova A.Kh., Aliev N.A., Solution of boundary value problem for Cauchy-Riemann equation in half-plane field./ Material's of XIV International scientific Kravchuk conference, Ukraine, Kiev, April 19-21,2012, pp.40-43. 